\title{
Evaluation of neonatal jaundice in the Makkah region
}

SUBJECT AREAS:

PAEDIATRIC RESEARCH

OXIDOREDUCTASES

Received

29 November 2013

Accepted

9 April 2014

Published

25 April 2014

Correspondence and requests for materials should be addressed to E.E.M.N.E. (emattia@ uqu.edu.sa)

\author{
Abdulaziz Alkhotani ${ }^{1}$, Essam Eldin Mohamed Nour Eldin ${ }^{2}$, Amal Zaghloul ${ }^{3} \&$ Shakil Mujahid ${ }^{4}$
}

'Department of Pediatrics, Faculty of Medicine, Umm Al-Qura University, Makkah, K.S.A., ${ }^{2}$ Department of Clinical Biochemistry, Faculty of Medicine, Umm Al-Qura University, Makkah, K.S.A., ${ }^{3}$ Department of Hematology and Immunology, Faculty of Medicine, Umm Al-Qura University, Makkah, K.S.A., ${ }^{4}$ Blood Bank, Ministry of Health, Makkah, K.S.A.

The aims of this study were to detect the frequency at which the different types of neonatal jaundice occur in Makkah and to estimate the malondialdehyde (MDA) levels. This study included 239 neonates with neonatal jaundice, 20 anemic neonates and 21 healthy neonates. ABO incompatibility was observed in $31.6 \%$ of neonates with indirect hyperbilirubinemia, in $14.3 \%$ of those with early onset jaundice, in $9.5 \%$ of those with persistent jaundice, in $8.5 \%$ of those with physiological jaundice, in $5 \%$ of anemic neonates and in $12 \%$ of all neonates. glucose-6-phosphate dehydrogenase (G6PD) deficiency was observed in $10.5 \%$ of neonates with indirect hyperbilirubinemia, in $3.9 \%$ of those with physiological jaundice, in $11.1 \%$ of those with direct hyperbilirubinemia, in $12 \%$ of those with persistent jaundice, in $10 \%$ of anemic neonates and in $6.6 \%$ of all neonates. $\mathrm{Rh}$ incompatibility and polycythemia were found in $2.6 \%$ of neonates with indirect hyperbilirubinemia and in $\mathbf{0 . 4 \%}$ of all neonates. In comparison to control group, MDA was significantly higher in all groups except for the anemic group. In conclusion, ABO incompatibility and G6PD deficiency frequently result in neonatal jaundice in Makkah, whereas $\mathrm{Rh}$ incompatibility and polycythemia are rare. The MDA level may serve as an indicator of oxidative stress.

eonatal jaundice is a benign condition that often does not require intervention. Most cases of neonatal hyperbilirubinemia constitute physiological jaundice and do not have serious consequences. An important clinical challenge is to identify those newborns who are at risk of developing severe neonatal hyperbilirubinemia ${ }^{1}$. There are many factors implicated in the development of pathological jaundice, including perinatal factors (e.g., birth trauma or infections), maternal factors(e.g., Rh or ABO incompatibility), neonatal factors (e.g., prematurity or polycythemia), and genetic factors (e.g., Crigler-Najjar's or Gilbert's syndrome) ${ }^{2}$. In addition, the administration of drugs such as cephalosporins and glucose-6-phosphate dehydrogenase (G6PD) enzyme deficiency have been implicated in pathological jaundice ${ }^{2}$. The mechanism by which hyperbilirubinemia occurs is through either increased bilirubin production (resulting from hemolysis, sepsis, blood extravasation or polycythemia) or increased enterohepatic circulation (resulting from prematurity, pyloric stenosis, delayed bacterial gut colonization, gastrointestinal tract immobility or obstruction), or decreased bilirubin elimination which occurs in Crigler-Najjar's and Gilbert's syndromes ${ }^{2}$.

$\mathrm{ABO}$ incompatibility usually occurs in the offspring of women with type O blood and occasionally in mothers with both type A blood and high anti-B IgG levels ${ }^{3}$. ABO incompatibility exists in approximately $15-25 \%$ of all maternal/fetal pairs. However, ABO hemolytic disease of the newborn occurs in approximately $1 \%$ of group $\mathrm{O}$ mothers with high antenatal IgG antibody titers ${ }^{4}$. Currently, the use of prophylactic anti-D immunoglobulin greatly reduces the incidence of hemolytic disease of the newborn due to Rh incompatibility ${ }^{5}$.

G6PD deficiency is an X-linked recessive disease that results in clinical manifestations such as neonatal jaundice, chronic nonspherocytic anemia, infection and drug-induced hemolysis ${ }^{6}$. A role for G6PD deficiency in the genesis of neonatal hyperbilirubinemia has been reported ${ }^{7}$. The most serious complication of G6PD deficiency in newborns is kernicterus resulting from severe neonatal hyperbilirubinemia.

Infants who experience chronic or acute fetal hypoxia have a higher incidence of polycythemia, with hematocrit levels greater than $65 \%$. Neonatal hyperbilirubinemia is a common clinical feature ${ }^{8}$.

Hyperbilirubinemia can also result from increased oxidative stress. It has been reported that bilirubin can function as a scavenger of reactive oxygen species ${ }^{9}$. Malondialdehyde (MDA) is a reactive metabolic product that results from the effects of reactive oxygen species on tissues and from a series of reactions that occur during lipid peroxidation ${ }^{10}$. Plasma MDA levels are a sensitive indicator of lipid peroxidation and thus of oxidative stress ${ }^{11,12}$. Therefore, measuring the MDA concentration in hyperbilirubinemic newborns will help determine the relationship between oxidative stress and elevated bilirubin levels. The aim of this study was to determine the frequency at 
which the different types of neonatal jaundice occur in the Makkah region. We also sought to determine the prevalence of $\mathrm{ABO}$ incompatibility, Rh incompatibility, G6PD deficiency, and polycythemia in neonates with different types of jaundice. Finally, we sought to estimate the levels of MDA and correlate them with the various parameters studied.

\section{Results}

This study included 239 jaundiced neonates, 20 anemic babies, and 21 healthy neonates (control group). The neonates consisted of 169 males and 111 females (a ratio of 3 male : 2 female). The numbers of neonates in each group was $\mathrm{G} 1, \mathrm{n}=21, \mathrm{G} 2, n=38, \mathrm{G} 3, n=129, \mathrm{G} 4$, $n=9, \mathrm{G} 5, n=42, \mathrm{G} 6, n=21$, and G7, $n=20$ neonates(Table 1 ). The total number of neonates with hyperbilirubinemia and anemia was 259. Of these neonates, 74 were preterm. The members of groups 2,3 , and 6 were significantly younger $(p<0.001)$ than those in the control group (G1). In contrast, those in group 7 were significantly older than those in G1 (Table 2). Six neonates (15.8\%) were preterm in G2, $37(28.7 \%)$ were preterm in G3, $1(11.1 \%)$ was preterm in $\mathrm{G} 4,15$ $(35.7 \%)$ were preterm in G5, 9 (42.9\%) were preterm in G6 and 6 (30\%) were preterm in G7. No significant differences were found with regard to age among preterm and term infants in any of the groups $(p>0.05)$.

Several parameters were evaluated for each group. The results are summarized in Table 2. Comparisons between the different groups were performed using the Kruskall-Wallis test. We found highly significant differences between the groups in age, total bilirubin levels, direct bilirubin levels, G6PD activity, red blood cell counts and hemoglobin concentrations $(p<0.001)$. No significant differences in MDA levels were found $(p>0.05)$.

As shown in Figure 1, the frequencies of neonatal jaundice are as follows: physiological jaundice-53.9\% (129/239), persistent jaundice$17.6 \%$ (42/239), indirect hyperbilirubinemia-15.9\% (38/239), early onset jaundice-8.8\% (21/239), and direct hyperbilirubinemia-3.8\% (9/239).

The highest prevalence of $\mathrm{ABO}$ incompatibility was found in the indirect hyperbilirubinemia group $(12 / 38,31.6 \%)$ followed by the early onset jaundice group $(3 / 21,14.3 \%)$, the persistent jaundice group $(4 / 42,9.5 \%)$, the physiological jaundice group $(11 / 129$, $8.5 \%)$ and the anemic group $(1 / 20,5 \%)$. The frequency of $\mathrm{ABO}$ incompatibility in all studied groups was $31 / 259$ (12\%) (Fig. 2). $\mathrm{ABO}$ incompatibility was determined by clinical classification of the mother as type $\mathrm{O}$ and the neonate as type $\mathrm{A}$ or $\mathrm{B}$.

Regarding hemoglobin concentrations in neonates with $\mathrm{ABO}$ incompatibility, no significant differences were observed between the control group and the indirect hyperbilirubinemia group, the physiological jaundice group, or the early onset jaundice group. However, the persistent jaundice group had significantly decreased hemoglobin levels than the control group $(11.0 \pm 1.4, p=0.01)$.
The frequency of $\mathrm{Rh}$ incompatibility was $1 / 38$ (2.6\%) in the indirect hyperbilirubinemia group. Rh incompatibility occurred in $0.4 \%$ of all neonates. The frequency of polycythemia was $2.6 \%(1 / 38)$ in the indirect hyperbilirubinemia group and $0.4 \%$ of all neonates. The direct Coombs' test was used in all cases. A positive result was found in only 5 cases of $\mathrm{ABO}$ incompatibility (16.1\%) and in only one case of Rh incompatibility.

The frequency of G6PD enzyme deficiency among the different groups is shown in Table 3. The classification of G6PD deficiency was performed according to the $\mathrm{WHO}^{17}$. No cases of class I G6PD deficiency were found. However, 10 cases of class $\mathrm{V}$ were observed. Of these, 2 were in G2 (one male and one female), 6 were in G3 (five males and 1 female), 2 were in G5 (both female), and 2 were in G6 (both female). No significant differences were found between males and females regarding G6PD concentrations, with the exception of G6, the early onset of jaundice, which showed significantly higher G6PD concentrations in the female group than in the male group ( $p$ $>0.05$ and $p<0.05$, respectively) (Table 4 ). There was no significant difference in the concentrations of hemoglobin between the neonates with G6PD deficiency and the control group. The hemoglobin concentrations in each group were $13.7 \pm 3.1$ and $15.3 \pm 2.0$, respectively.

Using the Spearman correlation, a significant negative correlation was found between MDA and hemoglobin levels in the persistent jaundice group $(r=-0.98, \mathrm{p}=0.01)$. No other significant correlations were detected.

\section{Discussion}

Neonatal hyperbilirubinemia is associated with a variety of conditions. Severe NH poses a direct risk of permanent neurological sequelae. The early identification of neonates who are at a greater risk of developing severe neonatal hyperbilirubinemia is of paramount importance to preventing brain damage ${ }^{17}$.

In this study, the type of jaundice found at the highest frequency was physiological jaundice (53.9\%) followed by persistent jaundice (17.6\%), indirect hyperbilirubinemia, early onset jaundice and direct hyperbilirubinemia (Fig. 1). These findings are in line with various national and international studies ${ }^{18,19}$. Physiological aspects that can contribute to neonatal hyperbilirubinemia include increased bilirubin production, less efficient hepatic conjugation, and enhanced bilirubin absorption by the enterohepatic circulation ${ }^{20}$. Bilirubin has been proposed as an effective antioxidant, and modest elevations of bilirubin may be beneficial in neonates ${ }^{21}$.

$\mathrm{ABO}$ incompatibility and G6PD deficiency were found to be frequent causes of neonatal hyperbilirubinemia in our study, as well as in other studies. G6PD deficiency is the most common human genetic enzymopathy, affecting over 200 million individuals worldwide. It is closely associated with neonatal jaundice, chronic nonspherocytic hemolytic anemia, favism and food- or drug-induced acute hemolytic anemia ${ }^{22}$. In this study, class II G6PD enzyme deficiency

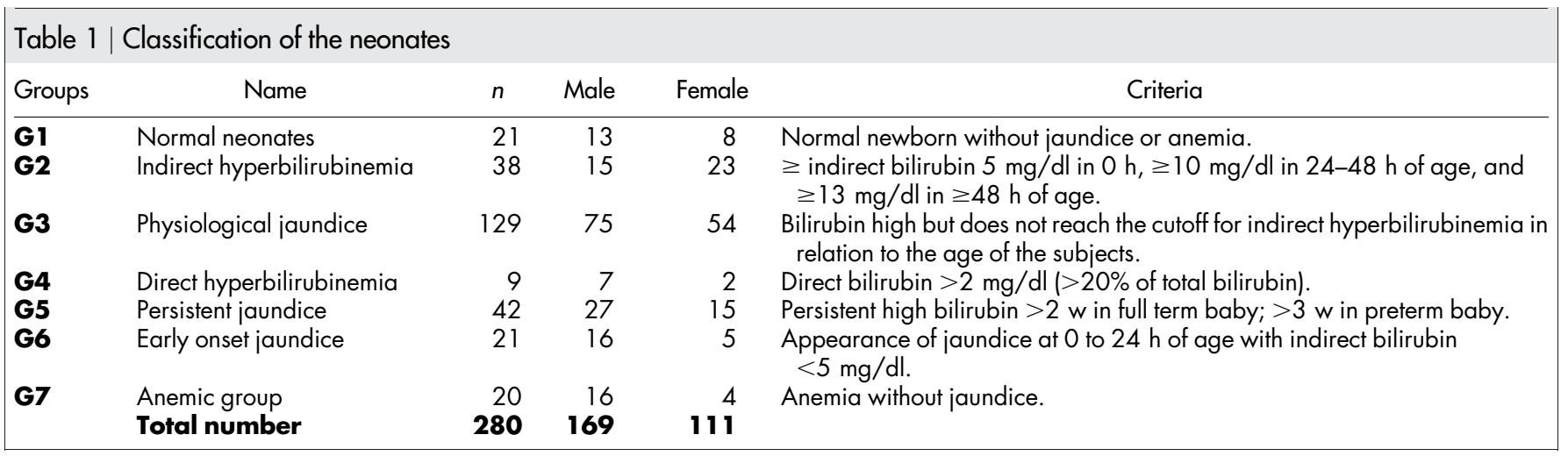



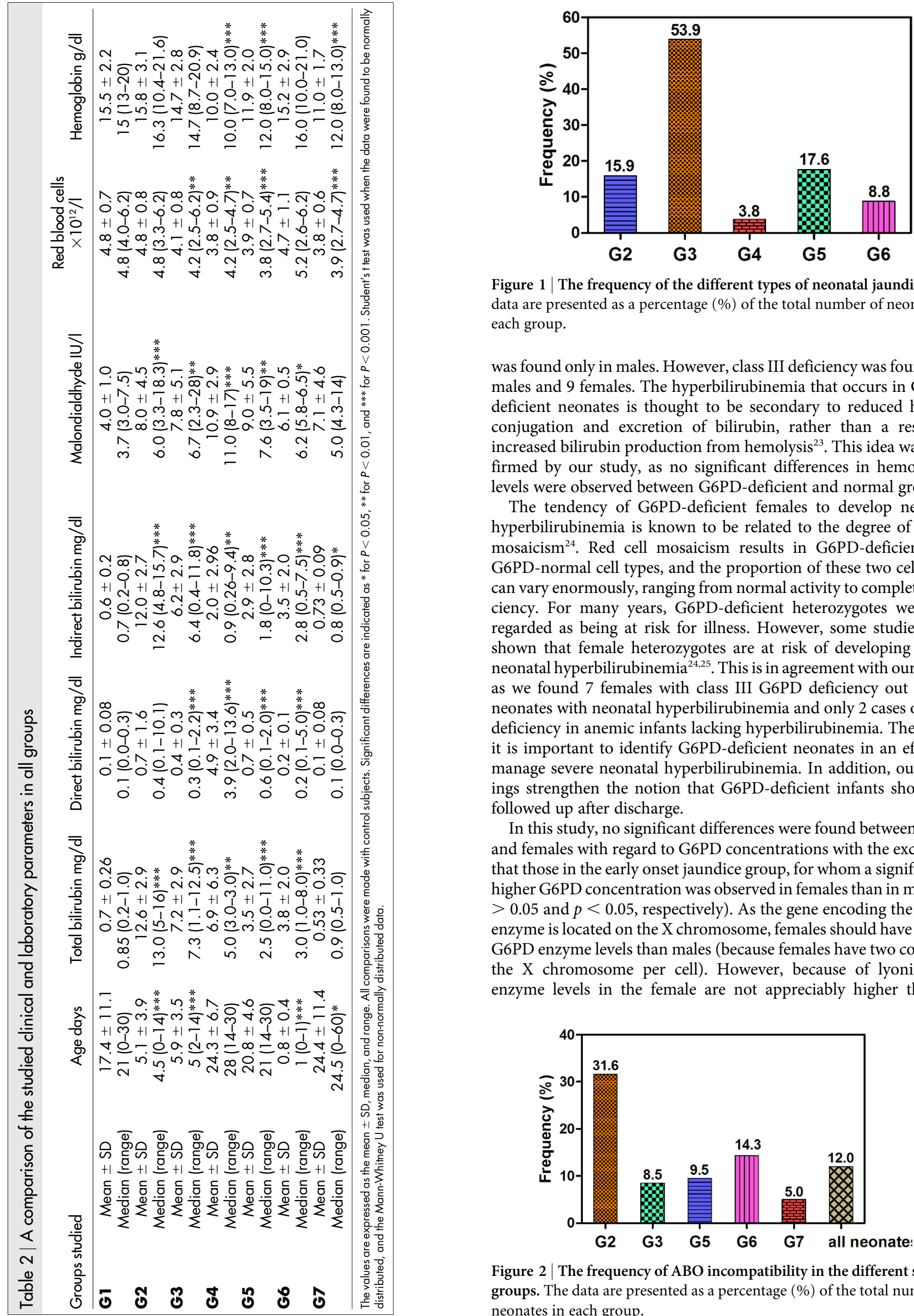

Figure $1 \mid$ The frequency of the different types of neonatal jaundice. The data are presented as a percentage (\%) of the total number of neonates in each group.

was found only in males. However, class III deficiency was found in 4 males and 9 females. The hyperbilirubinemia that occurs in G6PDdeficient neonates is thought to be secondary to reduced hepatic conjugation and excretion of bilirubin, rather than a result of increased bilirubin production from hemolysis ${ }^{23}$. This idea was confirmed by our study, as no significant differences in hemoglobin levels were observed between G6PD-deficient and normal groups.

The tendency of G6PD-deficient females to develop neonatal hyperbilirubinemia is known to be related to the degree of G6PD mosaicism $^{24}$. Red cell mosaicism results in G6PD-deficient and G6PD-normal cell types, and the proportion of these two cell types can vary enormously, ranging from normal activity to complete deficiency. For many years, G6PD-deficient heterozygotes were not regarded as being at risk for illness. However, some studies have shown that female heterozygotes are at risk of developing severe neonatal hyperbilirubinemia ${ }^{24,25}$. This is in agreement with our study, as we found 7 females with class III G6PD deficiency out of 111 neonates with neonatal hyperbilirubinemia and only 2 cases of such deficiency in anemic infants lacking hyperbilirubinemia. Therefore, it is important to identify G6PD-deficient neonates in an effort to manage severe neonatal hyperbilirubinemia. In addition, our findings strengthen the notion that G6PD-deficient infants should be followed up after discharge.

In this study, no significant differences were found between males and females with regard to G6PD concentrations with the exception that those in the early onset jaundice group, for whom a significantly higher G6PD concentration was observed in females than in males ( $p$ $>0.05$ and $p<0.05$, respectively). As the gene encoding the G6PD enzyme is located on the X chromosome, females should have higher G6PD enzyme levels than males (because females have two copies of the $\mathrm{X}$ chromosome per cell). However, because of lyonization, enzyme levels in the female are not appreciably higher than in

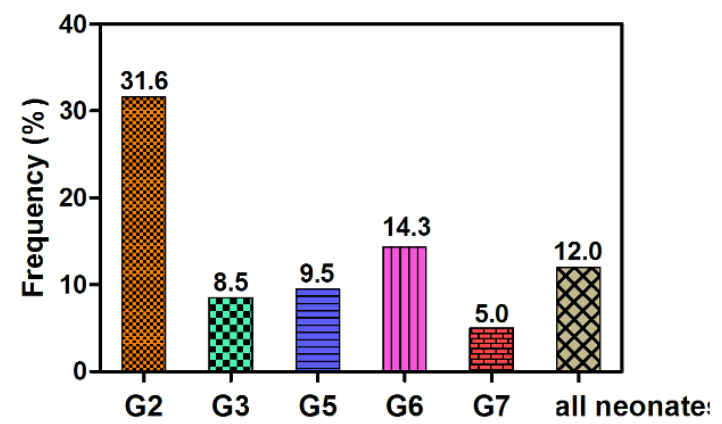

Figure $2 \mid$ The frequency of ABO incompatibility in the different studied groups. The data are presented as a percentage (\%) of the total number of neonates in each group. 
Table 3 | The frequency of G6PD enzyme deficiency in each group

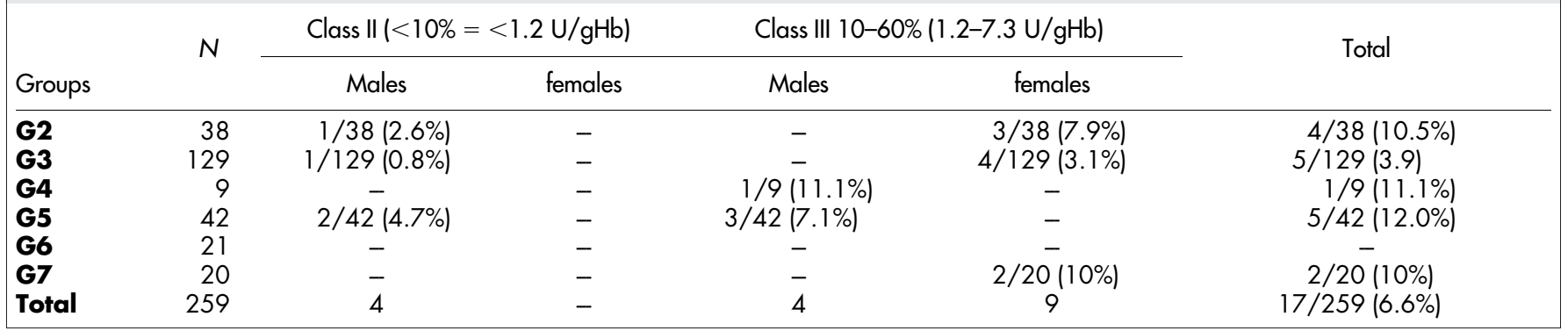

males $^{26}$. In those with early onset jaundice, 2 females had higher G6PD enzymatic activity (more than 150\% of the level of the control group). The remaining females had normal values. This could explain why, overall, females had significantly higher G6PD levels than did males.

This study is the first of its type performed in the Makkah region. We found that the frequency of G6PD enzyme deficiency in all studied neonates was $6.6 \%$. In previous national studies, the frequency of this deficiency was found to be $2 \%, 30.3 \%, 2 \%, 30.6 \%$, and $14.7 \%$ in $\mathrm{Yanbu}^{27}, \mathrm{Al}-\mathrm{Hofuf}^{28}$, Riyadh ${ }^{29}$, Al-Qatif ${ }^{30}$, and $\mathrm{Al}$ $\mathrm{Hasa}^{30}$, respectively. Studies from Iran ${ }^{31}, \mathrm{Brazi}^{32}$, and $\mathrm{India}^{33}$ found the frequency of G6PD deficiency to be $2.1 \%, 4.6 \%$, and $7.5 \%$, respectively. By contrast, studies from China ${ }^{34}$, Nigeria ${ }^{35}$, and Thailand $^{36}$ found higher frequencies of G6PD deficiency, at $18.42 \%, 25.5 \%$, and $38 \%$, respectively. In our study, $88.2 \%(15 / 17)$ of G6PD-deficient patients developed neonatal jaundice, of which $33.3 \%(5 / 15)$ were in the physiological jaundice group and the remaining cases had other types of jaundice. Our results indicate a higher frequency of jaundice in neonates with G6PD deficiency. Previous studies reported that one-third of children with G6PD deficiency develop neonatal jaundice ${ }^{22,37}$. Moreover, Tanphaichitr et al. (1995) reported that $49 \%$ of G6PD patients developed neonatal jaundice, of which $28.82 \%$ were classified as physiological and $20 \%$ as pathological ${ }^{38}$. The prevalence of the different subtypes of jaundice could explain the different presentations in different geographical locations worldwide.

Neonates with blood group A or B born to group O mothers are defined as having $\mathrm{ABO}$ incompatibility ${ }^{39}$. In our study, out of 259 neonates, $12 \%$ were $\mathrm{ABO}$ incompatible, which is in agreement with the national data ${ }^{28}$ but is lower than results of international studies $^{40,41}$. The direct Coombs' test was positive in only $16.1 \%$ cases, and the remaining cases were negative, which is similar to previous reports ${ }^{42,43}$. These reports showed that a positive direct Coombs' test in $\mathrm{ABO}$ incompatible neonates increases the risk of significant hyperbilirubinemia; however, when evaluated as a screening test for all newborns, the positive predictive value was found to be as low as $23 \%$ with readmission rates that were similar irrespective of the results from the Coombs' test in $\mathrm{ABO}$-incompatible children.

Table 4 | A comparison between males and females with regard to G6PD concentration $(\mathrm{U} / \mathrm{gHb})$ among the different groups

\begin{tabular}{lccl} 
Groups & Males Mean \pm SD & Females Mean \pm SD & $P$-value \\
\hline G1 & $12.0 \pm 2.5$ & $12.4 \pm 2.4$ & 0.828 \\
G2 & $14.5 \pm 6.6$ & $11.8 \pm 4.2$ & 0.203 \\
G3 & $13.7 \pm 4.8$ & $12.3 \pm 4.1$ & 0.192 \\
G4 & $9.5 \pm 4.0$ & $12.7 \pm 3.1$ & $0.498{ }^{\dagger}$ \\
G5 & $8.8 \pm 4.2$ & $13.8 \pm 6.8$ & 0.057 \\
G6 & $13.9 \pm 2.9$ & $20.7 \pm 0.9$ & $\mathbf{0 . 0 1 3}$ \\
G7 & $11.2 \pm 1.6$ & $9.6 \pm 4.4$ & 0.578 \\
\hline
\end{tabular}

Student's t test was used.

TThe Mann-Whitney $U$ test was used in these groups.
In most cases, $\mathrm{ABO}$ hemolytic disease of newborns causes hyperbilirubinemia without severe neonatal anemia, which is explained by the relatively few group A or B antigens on neonatal red blood cells and the presence of $A$ and $B$ antigens on other tissues and in body fluids $s^{44}$. This result was confirmed by our study, as most ABO incompatible cases had normal hemoglobin levels. Additionally, in our study, ABO incompatibility and G6PD were common causes of neonatal jaundice. If these are present, either phototherapy or intravenous immunoglobulin or both are administered to prevent severe jaundice and to decrease the need for exchange transfusion. Phototherapy was initiated based on the American Academy of Pediatrics phototherapy chart, whereas intravenous immunoglobulin was administered in $\mathrm{ABO}$ cases if the total bilirubin was two lines below the exchange transfusion line, in accordance with the exchange transfusion chart.

In the indirect hyperbilirubinemia group, one neonate presented with Rh hemolytic disease of the newborn (a frequency of $2.6 \%$ in the indirect hyperbilirubinemia group and $0.4 \%$ of all neonates). This finding is in agreement with previous studies, which state that $\mathrm{Rh}$ hemolytic disease of the newborn is less common than previously owing to the administration of $\mathrm{Rh}$ Ig, which results in a greater than $90 \%$ reduction in the alloimmunization rate among treated women ${ }^{41,45}$.

In this study, one neonate in the indirect hyperbilirubinemia group was polycythemic(2.6\%). Although polycythemia can reflect normal fetal adaptation, it has been thought to be responsible for abnormalities in neonates ${ }^{47}$.

MDA levels are a sensitive indicator of lipid peroxidation and thus of oxidative stress. Increased concentrations of free oxygen radicals in newborns damage the cell membrane through lipid peroxidation, and this damage may be associated with various pathologies such as hypoxic ischemic encephalopathy, intraventricular hemorrhage, necrotizing enterocolitis, and bronchopulmonary dysplasia ${ }^{46}$. Bilirubin is an effective scavenger of oxidant radicals, and its concentration is increased during oxidative stress. In this study, the MDA concentrations were significantly higher in all of the neonates with the various types of jaundice than in the control group (Table 2). In agreement with our results, Yigit et al (1999) found MDA concentrations in infants with hyperbilirubinemia to be remarkably $\operatorname{high}^{47}$. These MDA levels were thought to result from the response of the neonates to oxidative stress. It has been suggested that hyperbilirubinemia might play a defensive role against oxidative stress because the body's antioxidant mechanisms are not yet fully developed. It is known that biliary pigment can protect the cell membrane from lipid peroxidation ${ }^{48}$. In addition, some neonates in our study had infections, perinatal asphyxia, and respiratory distress syndrome, and approximately $30 \%$ of our cases were preterm. All of these conditions are considered to contribute to the elevation of MDA concentrations ${ }^{48-51}$.

In contrast to our results, Kumar et al (2007) found lower MDA levels in neonates with jaundice. This finding may arise from their study being conducted in neonates without high bilirubin level ${ }^{52}$. At higher bilirubin levels, the antioxidant effects of bilirubin might be 
negated by its direct cellular toxicity. In addition, Kumar et al excluded preterm neonates as well as those with infections, perinatal asphyxia, respiratory distress or major malformation, which were all included in our study. Furthermore, we found no significant correlation between bilirubin and MDA levels among the studied groups. This may indicate that factors other than MDA levels may contribute to the increased oxidative stress in our cases, such as stress and prematurity. Our results confirm those of Yigit et al (1998), who did not find a correlation between MDA levels and bilirubin concentrations in infants with non-hemolytic hyperbilirubinemia ${ }^{48}$. Moreover, we found a significant negative correlation between MDA and hemoglobin levels in the group with persistent jaundice. This may indicate that anemia accompanies many pathological processes in neonates and can negatively affect prognosis. Thus, increased MDA levels may indicate oxidative stress ${ }^{53}$.

One limitation of this study is that some factors that may have led to neonatal hyperbilirubinemia were not examined such as spherocytosis, thalassemia and sickle cell anemia.

Conclusions and recommendation. $\mathrm{ABO}$ incompatibility and G6PD deficiency are frequent causes of neonatal jaundice in Makkah, whereas Rh incompatibility and polycythemia are rarely observed. The MDA level could serve as an indicator of oxidative stress. Determinations of $\mathrm{ABO}$ and $\mathrm{Rh}$ incompatibility, G6PD deficiency and complete blood counts at birth are recommended to avoid serious complications.

\section{Methods}

Subjects. The protocol for this study was approved by the Umm Al-Qura University ethics committee. All participants gave informed consent in accordance with the declaration of Helsinki. The analytical methods were carried out in the research laboratory of the Biochemistry Department, Faculty of Medicine, Umm Al-Qura University in accordance with the approved guidelines.

This study was carried out from January 2011 to January 2013. All neonates were attended to at Alnour Hospital, Makkah, Saudi Arabia. The description and classification of all subjects is summarized in Table 1, with healthy neonates serving as a control group (G1). The jaundiced neonates were divided into 5 groups according to their indirect and direct bilirubin levels, the time of jaundice onset, and the persistence of jaundice ${ }^{13}$. Members of the indirect hyperbilirubinemia group (G2) were characterized as having increased levels of indirect bilirubin, where the concentration of indirect bilirubin was found to be $\geq 5 \mathrm{mg} / \mathrm{dl}$ at $0 \mathrm{~h}$ of age, $\geq 10 \mathrm{mg} / \mathrm{dl}$ at $24-48 \mathrm{~h}$ of age and $\geq 13 \mathrm{mg} / \mathrm{dl}$ at more than $48 \mathrm{~h}$ of age. In the physiological jaundice group (G3), the indirect bilirubin levels were not high enough to allow stratification based on age. In the direct hyperbilirubinemia group (G4), the levels of direct bilirubin were determined to be $>2 \mathrm{mg} / \mathrm{dl}$ ( $>20 \%$ of total bilirubin). Group 5 (G5) was composed of neonates with high bilirubin levels that had persistent jaundice for $>2$ weeks in full term neonates and $>3$ weeks in preterm neonates. Group 6 was composed of neonates with early onset jaundice (G6), in which jaundice was observed in the first $24 \mathrm{~h}$ of life but indirect bilirubin remained $<5 \mathrm{mg}$. Neonates in the anemic group (G7) were free of jaundice.

Sample collection. Four $\mathrm{ml}$ of blood was collected from neonates at the time of admission under aseptic conditions. $2 \mathrm{ml}$ of this blood was centrifuged for 15 minutes, and the serum was separated to allow MDA and total/direct bilirubin measurements. The remaining $2 \mathrm{ml}$ of blood was collected in EDTA tubes and used to perform blood counts, blood group analyses, G6PD enzyme assays, and direct antiglobulin tests. In addition, $1 \mathrm{ml}$ of blood was taken from each mother for blood group determination.

All participants were subjected to a:

1. Full clinical history.

2. Complete hemogram analysis using a Sysmex XT 2000i hematology analyzer (Sysmex Corporation of America, Long Grove, Illinois, USA), including red blood cells count, hemoglobin, packed cell volume, mean corpuscular hemoglobin, mean cell volume, mean corpuscular hemoglobin concentration, red cell distribution width, white blood cells count and platelet count.

3. ABO grouping of baby and mother (Dialab, Austria).

4. Direct antiglobulin test (Coombs' test) tube method. The reagent was supplied by Core Diagnostics LTD, Birmingham - United Kingdom.

5. Quantitative measurement of G6PD enzyme levels by the ultraviolet kinetic method (Trinity Biotech, USA) ${ }^{14}$.

6. MDA enzyme assay with the OxiSelect TBARS Assay Kit from Cell Biolabs, Inc. (USA) ${ }^{15}$.

7. Determination of direct and total bilirubin levels using the Dimension RxL max integrated chemistry system (Dade Behring, USA) ${ }^{16}$
Statistical analyses. All statistical analyses in this study were performed using the SPSS program (version 16). All quantitative data are described as the mean $\pm \mathrm{SD}$, the median, or the range. Comparisons between the control group and all other groups were performed using the Student's t test or the Mann-Whitney U test, depending on the distribution of the data. The Kruskal-Wallis $\mathrm{H}$ test was used when comparing all groups, as some of the data were not normally distributed. In addition, the Spearman correlation was used when correlating MDA with other parameters studied. Statistical significance was defined as $\mathrm{p}<0.05$.

1. Maisels, M. J. et al. Hyperbilirubinemia in the Newborn Infant $\geq 35$ Weeks Gestation: An Update With Clarifications. Pediatrics 124, 1193-1198, doi:10.1542/peds.2009-0329 (2009).

2. Dennery, P. A., Seidman, D. S. \& Stevenson, D. K. Neonatal Hyperbilirubinemia. N Engl J Med 344, 581-590, doi:10.1056/NEJM200102223440807 (2001).

3. Wang, M., Hays, T., Ambruso, D. R., Silliman, C. C. \& Dickey, W. C. Hemolytic disease of the newborn caused by a high titer anti-group B IgG from a group A mother. Pediatr Blood Cancer 45, 861-862, doi:10.1002/pbc.20503 (2005).

4. Chen, J. Y. \& Ling, U. P. Prediction of the development of neonatal hyperbilirubinemia in ABO incompatibility. Zhonghua Yi Xue Za Zhi 53, 13-18 (1994).

5. Roberts, I. A. G. The changing face of haemolytic disease of the newborn. Early Hum Dev 84, 515-523 (2008).

6. Singhi, S. C. Does nimesulide induce haemolysis in glucose-6-phosphate dehydrogenase deficiency? Acta Paediatr. 92, 637-638, doi:10.1111/j.16512227.2003.tb02524.x (2003).

7. Kaplan, M., Herschel, M., Hammerman, C., Hoyer, J. D. \& Stevenson, D. K. Hyperbilirubinemia Among African American, Glucose-6-Phosphate Dehydrogenase-Deficient Neonates. Pediatrics 114, e213-e219 (2004).

8. Sarkar, S. \& Rosenkrantz, T. S. Neonatal polycythemia and hyperviscosity. Semin Fetal Neonatal Med. 13, 248-255 (2008).

9. Saugstad, O. D. Free Radicals in Neonatal Intensive Care. In Tibboel, D. \& Voort, E. (Eds.), Intensive Care in Childhood. Update in Intensive Care and Emergency Medicine Vol. 25:36-52 (Springer Berlin Heidelberg, 1996).

10. Weinberger, B. et al. Association of Lipid Peroxidation with Antenatal Betamethasone and Oxygen Radical Disorders in Preterm Infants. Biol Neonate 85, 121-127 (2004)

11. Fulia, F. et al. Increased levels of malondialdehyde and nitrite/nitrate in the blood of asphyxiated newborns: reduction by melatonin. J Pineal Res. 31, 343-349, doi:10.1034/j.1600-079X.2001.310409.x (2001).

12. Gupta, P., Narang, M., Banerjee, B. \& Basu, S. Oxidative stress in term small for gestational age neonates born to undernourished mothers: a case control study. BMC Pediatrics 4, 14 (2004).

13. Gleason, C. A., Devaskar, S. U. \& Avery, M. E. Avery's diseases of the newborn/ [edited by] Christine A. Gleason, Sherin U. Devaskar. 9th edn, (Elsevier/Saunders, 2012).

14. Lohr, G. W. \& Waller, H. D. in Methods of Enzymatic Analysis (ed Bergmeyer, H. U.) 636-641 (Academic Press, 1974).

15. Ohkawa, H., Ohishi, N. \& Yagi, K. Assay for lipid peroxides in animal tissues by thiobarbituric acid reaction. Anal Biochem 95, 351-358 (1979)

16. Doumas, B. T., Wu, T. W. \& Jendrzejczak, B. Delta bilirubin: absorption spectra, molar absorptivity, and reactivity in the diazo reaction. Clin Chem 33, 769-774 (1987).

17. Cheng, S. W., Chiu, Y. W. \& Weng, Y. H. Etiological analyses of marked neonatal hyperbilirubinemia in a single institution in Taiwan. Chang Gung Med J 35, 148-154 (2012)

18. Bhutani, V. K., Johnson, L. H. \& Shapiro, S. M. Kernicterus in sick and preterm infants (1999-2002): a need for an effective preventive approach. Semin Perinatol 28, 319-325 (2004)

19. Abdel Fattah, M., Abdel Ghany, E., Adel, A., Mosallam, D. \& Kamal, S. Glucose-6phosphate dehydrogenase and red cell pyruvate kinase deficiency in neonatal jaundice cases in Egypt. Pediatr Hematol Oncol 27, 262-271, doi:doi:10.3109/ 08880011003639986 (2010).

20. Dennery, P. A., Seidman, D. S. \& Stevenson, D. K. Neonatal hyperbilirubinemia. N Engl J Med 344, 581-590, doi:10.1056/NEJM200102223440807 (2001).

21. Dennery, P. A., Weng, Y. H., Stevenson, D. K. \& Yang, G. The biology of bilirubin production. J Perinatol 21 Suppl 1, S17-20; discussion S35-19, doi:10.1038/ sj.jp.7210627 (2001).

22. Beutler, E. G6PD deficiency. Blood 84, 3613-3636 (1994).

23. Nabavizadeh, S. H., Rezaie, M., Sabzali, P., Barati, A. \& Zoladl, M. Cohort Study on Hemolysis Associated with G6PD Deficiency in Jaundice Neonates. Life Sci J 9, (2012).

24. Meloni, T., Forteleoni, G., Dore, A. \& Cutillo, S. Neonatal hyperbilirubinaemia in heterozygous giucose-6-phosphate dehydrogenase deficient females. $\mathrm{Br} \mathrm{J}$ Haematol. 53, 241-246 (1983).

25. Reclos, G., Hatzidakis, C. \& Schulpis, K. Glucose-6-phosphate dehydrogenase deficiency neonatal screening: preliminary evidence that a high percentage of partially deficient female neonates are missed during routine screening. J Med Screen 7, 46-51 (2000).

26. Obasa, T. O., Adesiyun, O. O., Mokuolu, O. A. \& Ojuawo, A. I. Comparative analysis of glucose-6-phosphate dehydrogenase levels in pre-term and term babies delivered at University of Ilorin Teaching Hospital. Pediatr Rep. 4, (2012). 
27. Muzaffer, M. A. Neonatal screening of glucose-6-phosphate dehydrogenase deficiency in Yanbu, Saudi Arabia. J Med Screen 12, 170-171, doi:10.1258/ 096914105775220660 (2005).

28. Al-Omran, A., Al-Ghazal, F., Gupta, S. \& John, T. B. Glucose-6-phosphate dehydrogenase deficiency and neonatal jaundice in Al-Hofuf area. Ann Saudi Med 19, 156-158 (1999).

29. Niazi, G. A., Adeyokunnu, A., Westwood, B. \& Beutler, E. Neonatal jaundice in Saudi newborns with G6PD Aures. Ann Trop Paediatr 16, 33-37 (1996).

30. Nasserullah, Z. et al. Neonatal screening for sickle cell disease, glucose-6phosphate dehydrogenase deficiency and a-thalassemia in Qatif and Al Hasa. Ann Saudi Med 18, 289-292 (1998).

31. Abolghasemi, H., Mehrani, H. \& Amid, A. An update on the prevalence of glucose-6-phosphate dehydrogenase deficiency and neonatal jaundice in Tehran neonates. Clin Biochem 37, 241-244, doi:10.1016/j.clinbiochem.2003.11.010 (2004).

32. Carvalho, C. G. et al. Glucose-6-phosphate-dehydrogenase deficiency and its correlation with other risk factors in jaundiced newborns in Southern Brazil. Asian Pac J Trop Biomed 1, 110-113, doi:10.1016/S2221-1691(11)60006-3 (2011).

33. Iranpour, R., Akbar, M. R. \& Haghshenas, I. Glucose-6-phosphate dehydrogenase deficiency in neonates. Indian J Pediatr 70, 855-857 (2003).

34. Weng, Y. H., Chou, Y. H. \& Lien, R. I. Hyperbilirubinemia in healthy neonates with glucose-6-phosphate dehydrogenase deficiency. Early Hum Dev 71, 129-136 (2003).

35. Uko, E. K., Agwunobi, S. N. \& Udoh, J. J. Glucose-6-phosphate dehydrogenase (G6-PD) levels in jaundiced neonates in Calabar. Niger J Med 12, 98-102 (2003).

36. Thaithumyanon, P. \& Visutiratmanee, C. Double phototherapy in jaundiced term infants with hemolysis. J Med Assoc Thai 85, 1176-1181 (2002).

37. Badens, C. et al. [Glucose-6-phosphate dehydrogenase et neonatal jaundice]. Presse Med 30, 524-526 (2001)

38. Tanphaichitr, V. S. et al. Glucose-6-phosphate dehydrogenase deficiency in the newborn: its prevalence and relation to neonatal jaundice. Southeast Asian J Trop Med Public Health 26 Suppl 1, 137-141 (1995).

39. Weng, Y. H. \& Chiu, Y. W. Spectrum and outcome analysis of marked neonatal hyperbilirubinemia with blood group incompatibility. Chang Gung Med J 32, 400-408 (2009).

40. Moerschel, S. K., Cianciaruso, L. B. \& Tracy, L. R. A practical approach to neonatal jaundice. Am Fam Physician 77, 1255-1262 (2008).

41. Khattak, I. D. et al. Frequency of ABO and Rhesus blood groups in District Swat, Pakistan. J Ayub Med Coll Abbottabad 20, 127-129 (2008).

42. Dinesh, D. Review of positive direct antiglobulin tests found on cord blood sampling. J Paediatr Child Health 41, 504-507, doi:10.1111/j.14401754.2005.00692.x (2005).

43. Madan, A., Huntsinger, K., Burgos, A. \& Benitz, W. E. Readmission for newborn jaundice: the value of the Coombs' test in predicting the need for phototherapy. Clin Pediatr (Phila) 43, 63-68 (2004).

44. Garratty, G. Hemolytic disease of the newborn. (American Association of Blood Banks, 1984).

45. Irshad, M. et al. Prevalence of Rhesus type and ABO incompatibility in jaundiced neonates. Vol. 25 (2011).

46. Kirimi, E. et al. Increased serum malondialdehyde level in neonates with hypoxicischaemic encephalopathy: prediction of disease severity. J Int Med Res 38, 220-226 (2010).
47. Yigit, S. et al. Serum malondialdehyde concentration in babies with hyperbilirubinaemia. Arch Dis Child Fetal Neonatal Ed 80, F235-237 (1999).

48. Yigit, S. et al. Serum malondialdehyde concentration as a measure of oxygen free radical damage in preterm infants. Turk J Pediatr 40, 177-183 (1998).

49. Kapoor, K., Basu, S., Das, B. K. \& Bhatia, B. D. Lipid peroxidation and antioxidants in neonatal septicemia. J Trop Pediatr 52, 372-375, doi:10.1093/tropej/fml013 (2006).

50. Mondal, N., Bhat, B. V., Banupriya, C. \& Koner, B. C. Oxidative stress in perinatal asphyxia in relation to outcome. Indian J Pediatr 77, 515-517, doi:10.1007/ s12098-010-0059-4 (2010).

51. Krediet, T. G., Kavelaars, A., Vreman, H. J., Heijnen, C. J. \& van Bel, F. Respiratory distress syndrome-associated inflammation is related to early but not late peri/ intraventricular hemorrhage in preterm infants. J Pediatr 148, 740-746, doi:10.1016/j.jpeds.2006.01.037 (2006)

52. Kumar, A., Pant, P., Basu, S., Rao, G. R. \& Khanna, H. D. Oxidative stress in neonatal hyperbilirubinemia. J Trop Pediatr 53, 69-71, doi:10.1093/tropej/fml060 (2007).

53. Gulbayzar, S. et al. Malondialdehyde level in the cord blood of newborn infants. Iran J Pediatr 21, 313-319 (2011).

\section{Acknowledgments}

This research project was supported and funded by the Institute of Scientific Research and Revival of Islamic Culture, Umm Al-Qura University, Makkah, KSA. Grant No. 431043109013.

\section{Author contributions}

A.E.: Design of the study, clinical selection, diagnosis and classification of the cases, writing and revising of the main manuscript text. E.E.N.E.: Design of the study, performance of biochemical laboratory investigations, preparing tables and figures, editing, styling, writing and revising of the main manuscript text. A.Z.: Design of the study, performance of hematological laboratory investigations, statistical analysis, writing and revising of the main manuscript text. S.M.: Performance of hematological laboratory investigations, writing and revising of the main manuscript text.

\section{Additional information}

Competing financial interests: The authors declare no competing financial interests.

How to cite this article: Alkhotani, A., Eldin, E.E.M.N., Zaghloul, A. \& Mujahid, S. Evaluation of neonatal jaundice in the Makkah region. Sci. Rep. 4, 4802; DOI:10.1038/ srep04802 (2014)

This work is licensed under a Creative Commons Attribution-NonCommercialShareAlike 3.0 Unported License. The images in this article are included in the article's Creative Commons license, unless indicated otherwise in the image credit; if the image is not included under the Creative Commons license, users will need to obtain permission from the license holder in order to reproduce the image. To view a copy of this license, visit http://creativecommons.org/licenses/by-nc-sa/3.0/ 